\title{
Microstrip filters based on open stubs and SIR for high frequency and ultra-wideband applications
}

\author{
Ş. Tahaİmeci*, Bilal Tütüncü**, Faruk Bešlija* and Lamija Herceg* \\ * Department of Electrical and Electronics Engineering, Faculty of Engineering, International University of Sarajevo, Hrasničkacesta \\ 15, 71210 Ilidža, Sarajevo, Bosnia and Herzegovina. \\ ** Department of Electrical and Electronics Engineering, Faculty of Engineering, Van Yüzüncü Yıl University, Van, Turkey. \\ **Corresponding Author: bilaltutuncu@yyyu.edu.tr
}

Submitted : 30/05/2020

Revised : :18/05/2021

Accepted : 12/07/2021

\begin{abstract}
This paper includes two new microstrip filter configurations for high frequency and ultra-wideband applications. The first proposed filter is a composition of four parallel open-circuited stubs connected by optimized fractal-structured microstrip line. The filter response is a combination of three passing regions, namely, low pass from $0.1 \mathrm{GHz}$ to $3 \mathrm{GHz}$, band-pass from $4.5 \mathrm{GHz}$ to $9 \mathrm{GHz}$, and high pass from $10.5 \mathrm{GHz}$ to $13 \mathrm{GHz}$, separated by two rejection regions from $3 \mathrm{GHz}$ to $4.5 \mathrm{GHz}$ and $9 \mathrm{GHz}$ to $10.5 \mathrm{GHz}$. Deep and sharp rejection regions reaching up to $-44.6 \mathrm{~dB}$ with $40 \%$ fractional bandwidth (FBW) are observed with a good electrical performance. Furthermore, with a comparative table, the advantages of this proposed BSF in terms of FBW, compactness, and insertion loss are compared with recently reported related studies. Secondly, a dualband band-pass filter implementing a stepped-impedance resonator (SIR) and a modified H-shaped structure is presented. This filter is designed to operate in a low pass region up to $3.58 \mathrm{GHz}$ and a band-pass region from 15.38 to $21.65 \mathrm{GHz}$, with a wide stopband region between 4.46 and $14.07 \mathrm{GHz}$. The simulated and measured results are in good agreement. Compared to its peers, the compact size and low price allow for a wide application of these filter configurations, while passing frequencies allow operation in the unlicensed frequency spectrum, which is popular for high-speed communication.
\end{abstract}

Keywords: Microstrip Filter; Band pass; Band stop; Open stubs; SIR.

\section{INTRODUCTION}

The progress in the field of wireless communications, especially in terms of the rapid growth of the WLAN standards, has created a need for the radio frequency (RF) systems that can offer a multiband performance at a compact design dimensions and a competitive manufacturing price. In recent years, multipleband filters have been proposed and exploited extensively as a key circuit block especially in dual-band wireless 
communication systems (Xu et al., 2019; Wei et al., 2016; Şahin et al., 2016; Xia et al.,2018; Lan et al., 2016; Hua and $\mathrm{Lu}, 2018$ ). These filters may be realized using the microstrip filter design. In order to achieve a multiband response within a domain of the microstrip filter design, there are several strategies that can be implemented. Firstly, it is possible to combine multiple single-band filters into a multiband filter (Chen, 2021; Yang et al., 2018). These single-band filters can either be cascaded (Sirci et al., 2019; Hung et al., 2020), embedded into each other (Zobeyri and Eskandari, 2018), or implemented by combining two sets of resonators with common input and output (Ren et al., 2018; Zhang et al., 2019). However, these methods appear consuming in terms of size and complexity of the filter as it would be required to have a filter whose size is equal to the size of two filters. Also, these filters would need to be put together carefully to maintain the desired performance of the designed multiband filter without interfering with the individual performance of each separate single-band filter. It is obvious that a single circuit operating in two designated bands is to be preferred over multiple circuits doing the same thing, due to its reduced dimensions and costs.

In this paper, two new configurations of ultra-wideband microstrip filters are proposed. Firstly, a band-stop filter consisting of four parallel open-circuited stubs connected by optimized fractal-structured microstrip line is adopted for the UWB filter design. The simulations are conducted in the range from $0.1 \mathrm{GHz}$ to $13 \mathrm{GHz}$, using method-of-moments based electromagnetic simulator Sonnet Suites, v16.51 (www.sonnetsoftware.com). The filter is designed to operate partially in the unlicensed frequency region and partially in the lower and higher frequency regions, having two rejection regions; one of which is the frequency spectrum of the growing $5 \mathrm{G}$ technology. The three passing regions occupy frequency bands from $0.1 \mathrm{GHz}$ to $3 \mathrm{GHz}$ (low-pass), from 4.5 $\mathrm{GHz}$ to $9 \mathrm{GHz}$ (band-pass), and from 10.5 to $13 \mathrm{GHz}$ (high-pass), while the two rejection regions occupy frequency bands from $3 \mathrm{GHz}$ to $4.5 \mathrm{GHz}$ and from $9 \mathrm{GHz}$ to $10.5 \mathrm{GHz}$. In this way, the current requirements of passband and sharp out-of-band rejections are satisfied. The stopband ensures fractional bandwidth (FBW) of $40 \%$, which satisfies the ultra-wideband requirements. After the simulations are performed, the model is fabricated on lossy FR4 substrate that is widely used due to its cost and availability. The filter size is relatively small and compact, having total area of $17 \mathrm{~mm} \times 17 \mathrm{~mm}$. The size and the performance are achieved due to the optimized configuration. The variations of the configuration and the changes in the response are investigated. Furthermore, with a comparative table, the advantages of this proposed BSF in terms of FBW, compactness, and insertion loss are compared with recently reported related studies. Secondly, a dual-band filter design with a low pass (LP) and a band-pass (BP) region is proposed, utilizing the stepped-impedance resonator (SIR) and a modified H-shaped structure. These filters are preferred over those composed out of multiple single-band filters, due to their reduced size (Lan et al., 2016), a good stopband performance (Sarkar et al., 2016), and simple structure (Chu et al., 2019). This filter relies on the SIR topology combined with the modified H-shape to achieve the desired LP and the BP response. This secondly proposed filter exhibits an LP response up to 3.58 $\mathrm{GHz}$ and a BP response from 14.38 to $21.65 \mathrm{GHz}$. It also provides a stopband region of at least $10 \mathrm{~dB}$ in the range of about $4 \mathrm{GHz}$ to $14.07 \mathrm{GHz}$ according to measurement results. Also, the ultra-wideband (UWB) characteristics of this filter are discussed. UWB technology has a great potential in the development of the various modern transmission systems, for instance, through-wall imaging, medical imaging, vehicular radar, indoor, hand-held UWB systems, and others (Martin et al., 2018). For this filter, only UWB characteristics are observed, and not the regional limitations, such as those proposed by the FFC (Federal Communications Commission) for United States, MIIT (Ministry of Industry and Information Technology) for China, MinComSvyaz Rossi (Ministry of Digital Development, Communications and Mass Media of the Russian Federation) for Russia, and so on. Both filters proposed in this study are very compact and realized on a lowcost substrate, satisfying both size and production cost preferences. All proposed results are later discussed and verified via simulation and experiment. 


\section{BAND-STOP FILTER WITH OPEN STUBS}

\section{Material and Method}

The design methodology of this proposed filter begins with a conventional open-stub band-stop filter (BSF). A conventional model consists of two open stubs adjusted onto a microstrip transmission line. The length and the separation between the two stubs are quarter of the wavelength at the mid-band frequency (Shrestha and Kim, 2015; Crnojević-Bengin, 2015). This topology has been adopted for its simple structure (avoiding formations that would require complicated manufacturing processes that could provoke errors during measurements, like via holes for short-circuit stubs and backside slots for coplanar waveguide (CPW)).

As the design of the open stub filter is based on the electromagnetic field theory, the input impedance of the transmission line is given by the following:

$$
Z_{\text {in }}=Z_{0}\left(\frac{Z_{L} \cos \beta l+j Z_{0} \sin \beta l}{Z_{0} \cos \beta l+j Z_{L} \sin \beta l}\right)=Z_{0}\left(\frac{Z_{L}+j Z_{0} \tan \beta l}{Z_{0}+j Z_{L} \tan \beta l}\right)
$$

where $Z_{0}$ is the characteristic impedance, $Z_{\text {in }}$ and $Z_{\mathrm{L}}$ are the input and output impedances, respectively, $\beta$ is the propagation constant, and $l$ is the equivalent electrical length of the transmission lines. Furthermore, we have the guided wave length of the microstrip transmission line which can be calculated using Equation (2):

$$
\lambda_{g}=\frac{\lambda_{0}}{\sqrt{\epsilon_{\text {eff }}}}=\frac{c}{f_{b s} \sqrt{\epsilon_{\text {eff }}}}
$$

where $\lambda_{0}$ is the wavelength in free-space, $\mathrm{c}$ is the speed of light $\left(3 \times 10^{8} \mathrm{~m} / \mathrm{s}\right)$, fbs is the center frequency of the BSF, and $\epsilon_{e f f}$ is the effective permittivity. The electrical length of the microstrip transmission line, approximately $\lambda_{1} / 2$, can be calculated by Equation (3):

$$
\theta_{1}=\beta l=\frac{2 \pi}{\lambda_{1}} \cdot \frac{\lambda_{0}}{2}
$$

In an identical way, the electrical lengths of the open stubs (also approximately having a length of $\lambda_{2} / 4$ ) can be calculated by Equation (4):

$\theta_{2}=\beta l=\frac{2 \pi}{\lambda_{2}} \cdot \frac{\lambda_{0}}{4}$

From Equations (1) and (3), we can write the following:

$$
Z_{\text {in }}=\frac{\left(Z_{0}\right)^{2}}{Z_{L}}
$$

Thus, if $Z_{L}$ is zero for the open stubs, then $Z_{\text {in }}$ is infinity in Equation (5), and using Equations (3) and (4), we can calculate the electrical length of the microstrip transmission lines (Martin et al., 2018). In this study, the 
conventional open-stub BSF is modified so that the satisfying response would be obtained for the demand of the popular UWB technology.

\section{Configuration and Response of the Proposed Filter}

The middle microstrip line (without stubs) is the one that generates LP filter with very high cut-off frequency around $10 \mathrm{GHz}$. When the stubs are added, they provoke the rejection band to appear in the LP region, which, in total, ends up being band-stop filter response. As already mentioned, the height of the open stubs, as well as the distance between them is related to the wavelength at the mid-band frequency. Such sizes would form much bigger filter box size. Introducing the application of fractal shape on the main transmission line, the distance between the four open stubs shortens, so the initial filter size can be reduced (Lan et al., 2016).

The schematic of the BSF is designed in Sonnet Suites, v16.51 (www.sonnetsoftware.com), to generate a simulation. Many trials led to the final design of the UWB filter that consists of four open stubs mounted on the microstrip line with a number of slits, that is, in the shape of a fractal curve. All practical microstrip circuits contain discontinuities. Types of microstrip discontinuities applied in this design are symmetrical steps in width and open ends. Conventionally, by attaching more open stubs onto a microstrip line, wider rejection bandwidth and deeper rejection can be achieved. The generated drawbacks of this are high insertion loss in the passing region and the increased size of the filter itself (Majidifar and Hayati, 2017). However, it is possible to regulate the response and keep the desired characteristics. The optimized configuration of the proposed BSF with open stubs and its fabricated prototype can be seen in Figure 1.
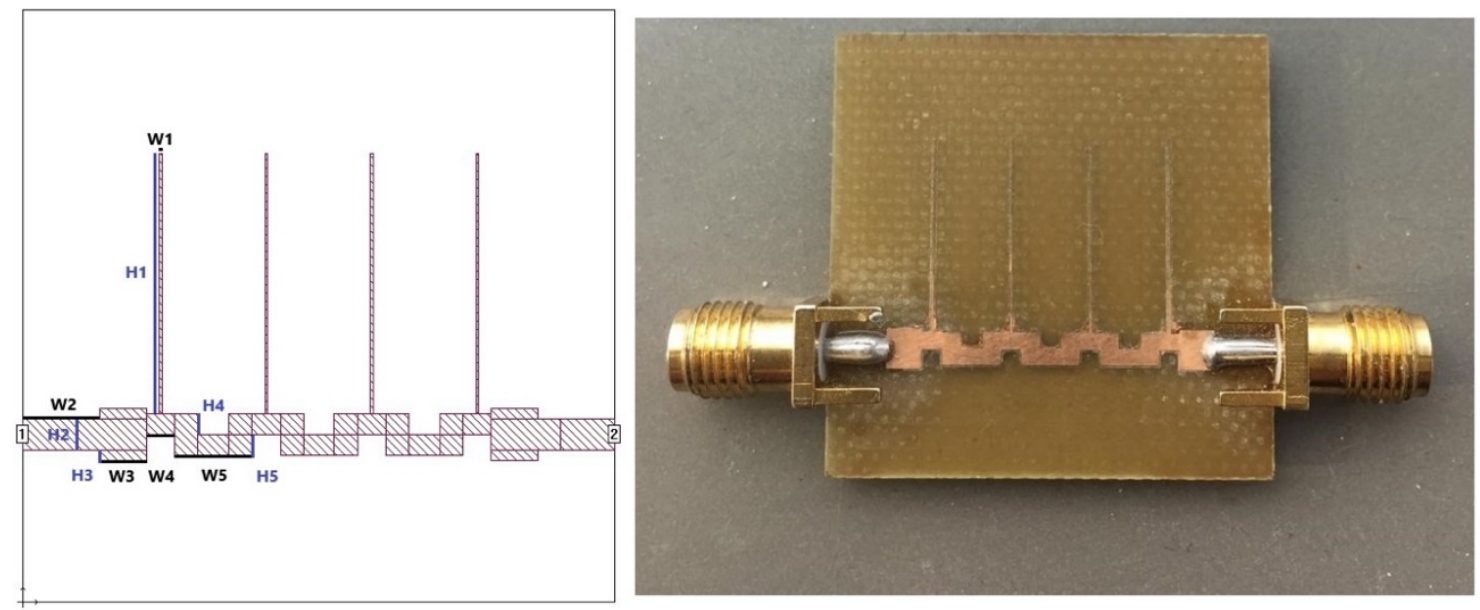

Figure 1. The configuration (left) and fabricated prototype (right) of the proposed BSF.

The filter box is small and compact with the total size of $16.96 \mathrm{~mm} \times 16.96 \mathrm{~mm}$. The exact dimensions of the configuration expressed in millimeters are $\mathrm{H} 1=7.827, \mathrm{H} 2=\mathrm{W} 4=0.8, \mathrm{H} 3=0.308, \mathrm{H} 4=\mathrm{H} 5=0.6, \mathrm{~W} 1=0.127$, $\mathrm{W} 2=\mathrm{W} 5=2.31$, and $\mathrm{W} 3=1.08$. The model is symmetrical, and the sizes are repeatable. The narrowest part is the width of the stubs that are 5 mils or $0.127 \mathrm{~mm}$, which is still permissible in terms of fabrication.

The prototype of the proposed BSF model is fabricated on FR4 substrate with a relative dielectric constant of $\varepsilon r=4.4$. Even though the losses in FR4 increase as the frequency gets higher, its low cost and availability make it preferable. The thickness of the substrate is $\mathrm{h}=1 \mathrm{~mm}$. The whole backside of the substrate (the ground 
plane) is copper-coated. The model is formed to be multiband band-stop filter, suppressing more than one signal band. Hence, three passing regions and two rejection regions are maintained in the simulated range from 0.1 $\mathrm{GHz}$ to $13 \mathrm{GHz}$. The filter is the two-port device, whose response is presented in terms of $\mathrm{S}$ parameters: $\mathrm{S}_{11}$ as the input port voltage reflection coefficient and $S_{21}$ as the forward voltage gain (Tütüncü, 2020). The filter response according to simulated and measured results can be seen in Figure 2.

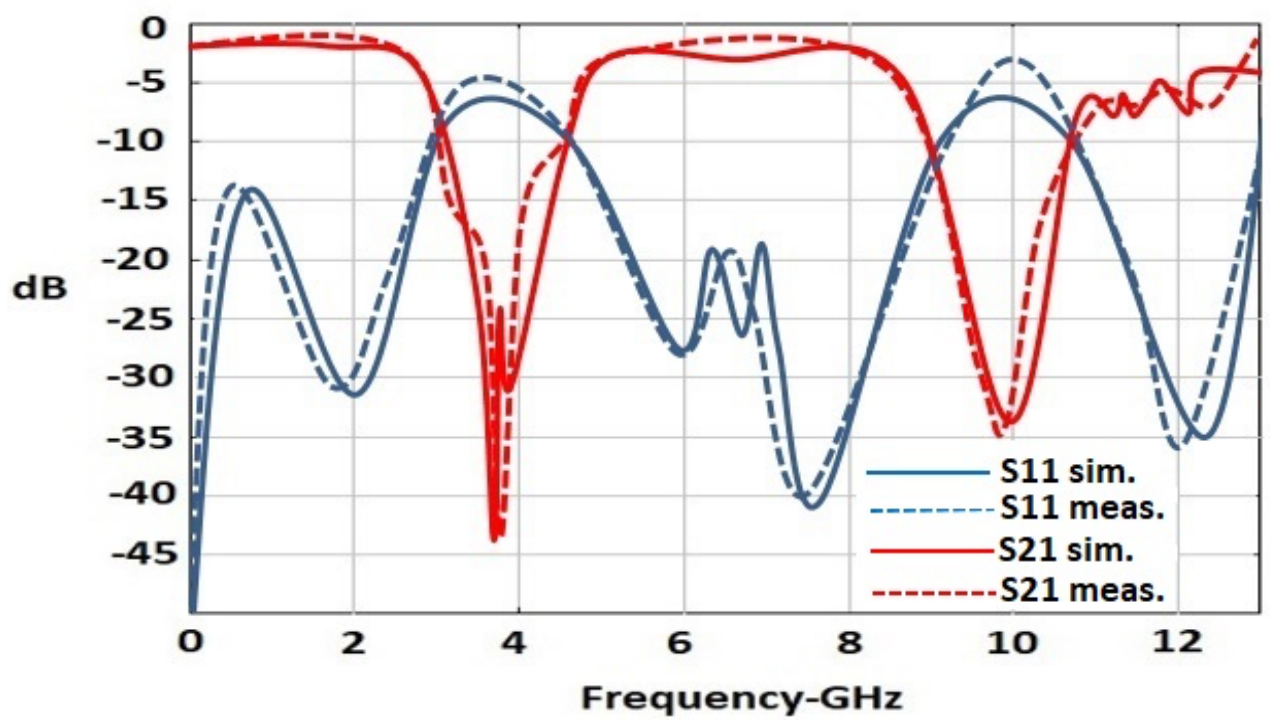

Figure 2. Frequency response of the proposed BSF with open stubs.

The passband covers from $0.1 \mathrm{GHz}$ to $3 \mathrm{GHz}$, from 4.5 to $9 \mathrm{GHz}$, and from $10.5 \mathrm{GHz}$ to $13 \mathrm{GHz}$, with the insertion losses between -0.3 and $-1.5 \mathrm{~dB}$, including connector losses. The simulated input matches are mainly $10 \mathrm{~dB}$ throughout the passing regions, being at most $-8 \mathrm{~dB}$. The rejection regions suppress frequencies from 3 $\mathrm{GHz}$ to $4.5 \mathrm{GHz}$ and from $9 \mathrm{GHz}$ to $10.5 \mathrm{GHz}$, and these regions are very sharp, reaching $-44.6 \mathrm{~dB}$ and $-35 \mathrm{~dB}$, respectively. The line impedance of the BSFs is $50 \Omega$. The simulated and measured responses of this filter ensured that a FBW of $40 \%$ is obtained in the lower rejection region. That is way bigger bandwidth than the 20 $\%$ usually attained by wideband filters or $50 \%$ usually attained by ultra-wideband filters. FBW is calculated according to Equation (6):

$$
F B W=\frac{f_{2}-f_{1}}{f_{c}}=\frac{f_{2}-f_{1}}{\frac{f_{2}+f_{1}}{2}}=\frac{4.5 \mathrm{GHz}-3 \mathrm{GHz}}{3.75 \mathrm{GHz}}=40 \%
$$

The measured results agree with the simulated results. The difference can be noticed in the insertion losses, especially in the middle passing region after $5 \mathrm{GHz}$, due to the lossy structure of FR4. In spite of that, the response still satisfies the design specifications. In order to increase the performance, the change in the configuration can be made.

\section{Configuration Variations of the BSF}

In this section, the adjustment of the filter performance by varying the parts of the configuration is discussed to strengthen the implementation of the proposed design for practical applications. Variations in the 
size of the open stubs, as well as in the main transmission line, result in attractive responses. Some of the changes in the BSF structure may involve the height and width of the open stubs, the size of the slits, the size of the main transmission line, number of the open stubs, and so on.

The height of open stubs is a critical factor affecting the frequency of the band-pass region. If they are shorter, band-pass region moves to the right. That is, it occupies higher frequencies. Longer stubs decrease the width of the rejection region, while shorter stubs increase the width of the passing region. The width of the open stubs affects the width of the band-stop region. Wider open stubs result in wider rejection bandwidth. Bigger slits affect the input matches by decreasing them. Wider lines next to the ports increase the attenuation considerably. However, they increase the losses and then disagree with the set requirements. The measured input matches can be notably improved when the size of the slits is modified in the configuration, that is, increased. That variation could be considered for the general improvement of the filter response. The other way of improving the input matches is increasing the width of the stubs, but the rejection region increases in that case, which would disagree with the requirements of sharp out-of-band rejection regions. In conclusion, the filter configuration is very sensitive because small changes in dimensions cause large changes in the filter response.

\section{Comparisons of Previous Studies with Proposed BSF}

Table 1 demonstrates the performance comparisons of the proposed BSF with some previously reported works, and it can be seen that our work has advantages over others in terms of overall size, FBW, and depth in insertion loss.

Table 1. Comparisons of previous studies with proposed BSF with open stubs.

\begin{tabular}{|c|c|c|c|c|}
\hline Ref. & $\mathbf{f}_{\mathbf{0}}$ & FBW & $\begin{array}{c}\text { Insertion } \\
\text { loss }\end{array}$ & 2D size $/ \boldsymbol{\lambda}^{\mathbf{2}}$ \\
\hline (Naglich and Guyette, 2015) & $3 \mathrm{GHz}$ & $\% 1.41$ & $30 \mathrm{~dB}$ & $1.14 \times 0.58$ \\
\hline (Guyette and Naglich, 2016) & $0.5 \mathrm{GHz}$ & $\% 6.66$ & $38 \mathrm{~dB}$ & $0.17 \times 0.14$ \\
\hline (Zuo and Yu, 2017) & $1 \mathrm{GHz}$ & $\mathrm{NA}$ & $40 \mathrm{~dB}$ & $0.27 \times 0.36$ \\
\hline (Karpuz et al., 2016) & $2.79 \mathrm{GHz}$ & $\% 4.04$ & $15.5 \mathrm{~dB}$ & $0.16 \times 0.22$ \\
\hline (Koirala et al., 2016) & $4.7 \mathrm{GHz}$ & $\% 31.02$ & $35.1 \mathrm{~dB}$ & $0.38 \times 0.07$ \\
\hline This work & $3.75 \mathrm{GHz}$ & $\% 40.0$ & $44.6 \mathrm{~dB}$ & $0.18 \times 0.18$ \\
\hline
\end{tabular}

\section{DUAL-BAND FILTER UTILIZING SIR}

In this section, a dual-band filter obtained by implementing SIR and a modified H-shaped structure is presented. This filter is designed to operate in two bands, namely, in a LP region and a BP region with a wide stopband region between 4.46 and $14.07 \mathrm{GHz}$. 


\section{Fundamental Resonance Properties of SIR}

The conditions of fundamental and spurious resonance of the SIR are presented in this Section and Figure 3 shows two typical SIR structures to be discussed. $Z_{1}$ and $Z_{2}$ are two different characteristic impedance lines, of admittance $Y_{1}$, and $Y_{2}$ of the symmetric SIR structure.
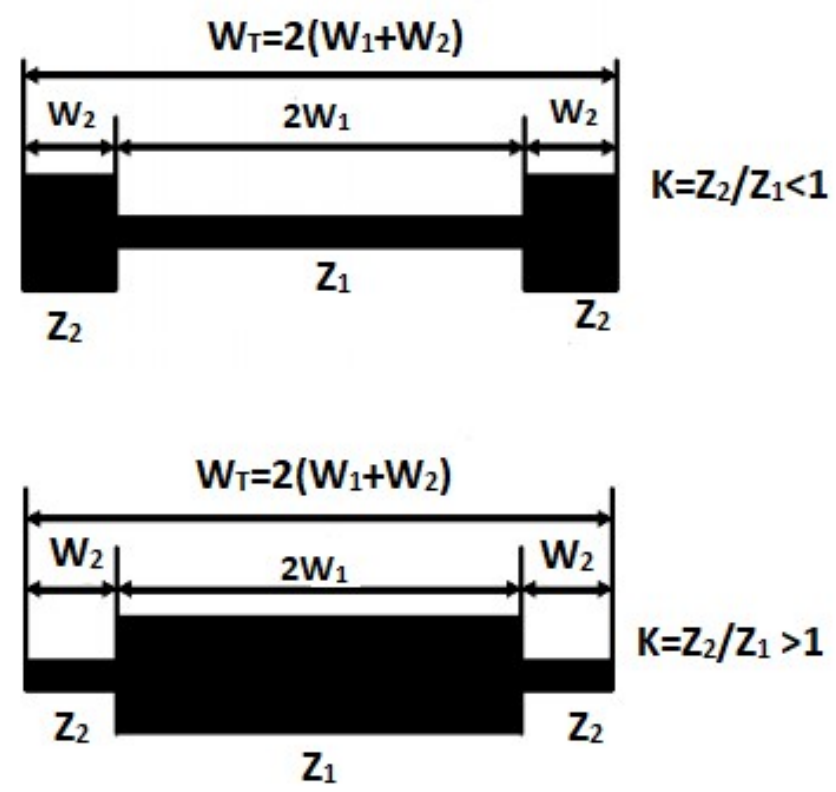

Figure 3. Typical SIR structure.

The admittance of the resonator from the open end, Yi is given as follows (Cameron et al., 2018):

$$
Y_{i}=j Y_{2} \frac{2\left(K \tan W_{1}+\tan W_{2}\right) \cdot\left(K-\tan W_{1} \tan W_{2}\right)}{K\left(1-\tan ^{2} W_{1}\right) \cdot\left(1-\tan ^{2} W_{2}\right)-2\left(1+K^{2}\right) \tan W_{1} \tan _{2}}
$$

Herein, the impedance ratio $K=Z_{2} / Z_{1}$ and $Y_{2}=1 / Z_{2}$. The resonance condition can be obtained when $Y_{i}=0$, and thus the fundamental resonance condition can be expressed as

$$
\mathrm{K}=\tan \mathrm{W} 1 \cdot \tan \mathrm{W} 2
$$

Thus, the relationship between $\mathrm{W}_{\mathrm{T}}$ and $\mathrm{W}_{1}$ is derived from (8) as

$$
\tan \frac{W_{T}}{2}=\frac{1}{1-K} \cdot\left(\frac{K}{\tan W_{1}}+\tan W_{1}\right)
$$

It is preferable to choose $\mathrm{W}_{1}=\mathrm{W}_{2}$ because the design equation can be simplified considerably for a practical application. Therefore, the SIR is treated as having $\mathrm{W}_{1}=\mathrm{W}_{2}=\mathrm{W}_{0}=\tan ^{-1} \sqrt{K}$. Consequently, taking the 
spurious resonance frequency to be $\mathrm{f}_{\mathrm{sn}}(\mathrm{n}=1,2,3 \ldots)$ and corresponding $\mathrm{W}$ with $\mathrm{W}_{\mathrm{sn}}(\mathrm{n}=1,2,3 \ldots)$ and $\mathrm{Y}_{\mathrm{i}}=0$, we obtain

$$
\tan \mathrm{W}_{\mathrm{s} 1}=0, \tan ^{2} \mathrm{~W}_{\mathrm{s} 2}-\mathrm{K}=0, \tan \mathrm{W}_{\mathrm{s} 3}=0 .
$$

Then,

$$
\begin{aligned}
& \frac{f_{s 1}}{f_{0}}=\frac{W_{s 1}}{W_{0}}=\frac{\pi}{2 \tan ^{-1} \sqrt{K}} \\
& \frac{f_{s 2}}{f_{0}}=\frac{W_{s 2}}{W_{0}}=2\left(\frac{f_{s 1}}{f_{0}}\right)-1 \\
& \frac{f_{s 3}}{f_{0}}=\frac{W_{s 3}}{W_{0}}=2\left(\frac{f_{s 1}}{f_{0}}\right)
\end{aligned}
$$

The above results are the function of the impedance ratio Kas detailed in Cameron et al, 2018. It becomes evident that the spurious response can be controlled by the impedance ratio $\mathrm{K}$, and this is one of the special features of the SIR.

\section{Physical Structure and Dimensions of the Proposed SIR Filter}

Design and simulation are performed at Sonnet Suites, version 16.53 (www.sonnetsoftware.com). In the simulation process, the relative permeability and thickness of the filter layers are adjusted according to the manufacturing capabilities of laboratory in which the filter is produced. For dielectric layer, FR-4 is used, with $1 \mathrm{~mm}$ thickness and $\mathrm{\varepsilon r}=4.4$.

Knowing that the analysis for the filter is conducted on a spectrum from 0 to $25 \mathrm{GHz}$, there is a high possibility of an undesired response at the certain frequencies for such a wide frequency span. One of the parameter that can alter the filter response in such way is the box resonance, which can be estimated by the simulation powers of Sonnet. Therefore, the box dimensions of the filter must be carefully selected. In order to prevent the box resonance, it is possible to select the box dimensions in such way that the box resonance frequencies are eliminated. Experimentally, it has been found that the ratio that causes the least number of box resonance frequencies is the golden ratio. The dimensions of the box are minimized with respect to this ratio, until there are no estimated box resonance frequencies, which happened for $10.2 \mathrm{~mm}$ width by $6.3 \mathrm{~mm}$ height. The additional $3 \mathrm{~mm}$ is added to each side to ease the soldering, resulting in a final dimension of the box of 16.2 $\mathrm{mm}$ width by $6.3 \mathrm{~mm}$ height.

After the filter box design, the main part of the filter structure would ensure that the desired dual-band response is considered as mentioned in the previous section. Starting with the SIR as an initial element (refer to Figure 4 as H1, H2, W1, and W2), adding a rectangular metallization centered on both sides would result in some multiband response, where one of the bands would be an LP. The H-shaped structure (refer to Figure 4 as 
$\mathrm{H} 5, \mathrm{H} 7$, and W4) serves here both as a single-band band-pss filter (BPF) and a tool to improve the rejection in the frequency range between the operating regions of the Low Pass Filter (LPF) and the BPF. Although this design ensures the desired cut-off frequencies for both regions, as a result of an $S_{11}$ parameter value around -6 $\mathrm{dB}$, the input match at the BP region is very poor. This issue requires design modifications which would both improve the downfall in $\mathrm{S}_{11}$ for $\mathrm{BP}$ region and preserve the current response at the other regions. Therefore, the $\mathrm{H}$-shaped structure is adjusted, so that the rejection in the region between the LPF and the BPF is preserved as much as possible, while the input match at the $\mathrm{BP}$ region is improved, so that an $\mathrm{S}_{11}$ parameter value is at least $10 \mathrm{~dB}$. This is achieved by introducing the discontinuities at the edges of the H-shape, namely, four equal slits on the inner sides of the horizontal metallization (refer to Figure 4 as H4, H5). The abovementioned adjustment results in both preserved response of the other regions and the input-match improvement of the BP region. It also introduces the shift of the BP region to the left (to lower cut-off frequencies). This indicates that the BP region cut-off frequencies may be adjusted by changing the height of this slit.

Finally, the filter is simulated in a very small step to see if there is an undesirable frequency response. As a result of multiple trials, two additional elements are added to improve these downfalls: 4 additional small boxes (refer to Figure 4 as H3, W5) to correct the randomly picked frequencies of the higher spectrum and 4 additional vertical lines ("fangs", refer to Figure 4 as H6, W7) to improve the transition of the response at the lower corner frequency of the BP region. The final version of the modified filter with SIR and its manufactured prototype are shown in Figure 4. The dimensions of the proposed filter are as follows: $\mathrm{W} 1=3, \mathrm{~W} 2=10.3$, $\mathrm{W} 3=\mathrm{H} 2=0.3, \mathrm{~W} 4=6.1, \mathrm{~W} 5=0.4, \mathrm{~W} 6=\mathrm{H} 5=0.5, \mathrm{~W} 7=0.2, \mathrm{~W} 8=2.4, \mathrm{~W} 9=\mathrm{H} 6=1, \mathrm{H} 1=0.9, \mathrm{H} 3=0.6, \mathrm{H} 4=1.2$, and $\mathrm{H} 7=4.3$ (all in $\mathrm{mm}$ ).
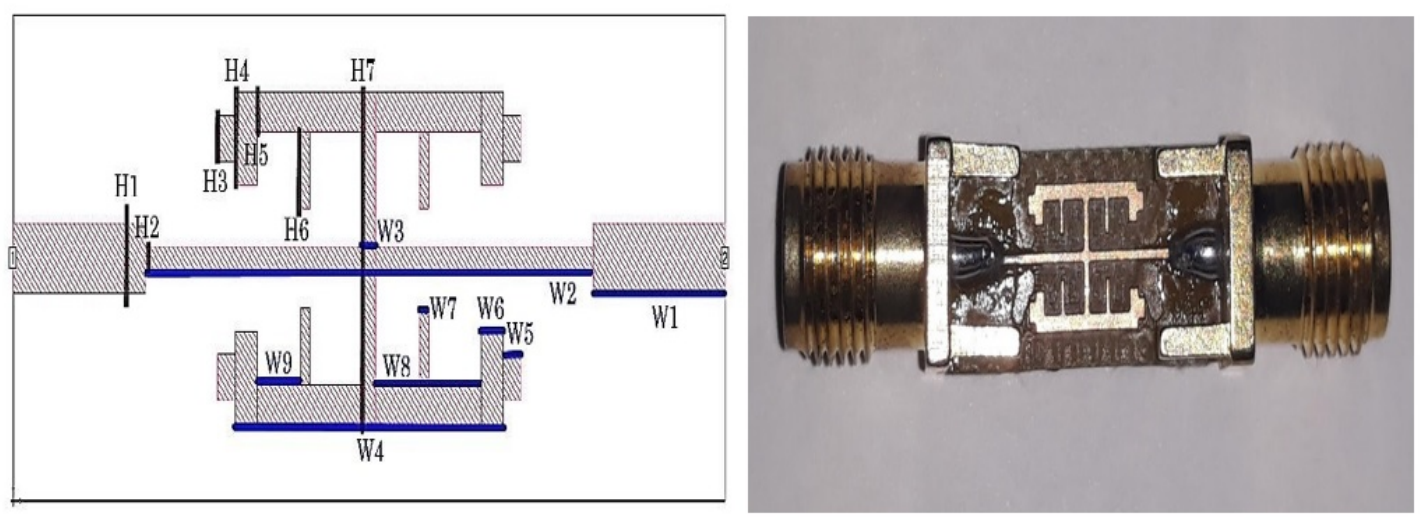

Figure 4. The schematic (left) and prototype (right) view of the proposed SIR filter.

\section{Simulation and Experimental Results}

The simulation results suggest that the filter exhibits the prescribed behavior in terms of the corner frequencies (namely, an LP response up to $3.58 \mathrm{GHz}$ and a BP response from 14.38 to $21.65 \mathrm{GHz}$ ), the bandwidths, and the rejections. The experimental results comply with the simulation results. Although there is no loss according to simulation results, the experimental results show a loss of up to $1.5 \mathrm{~dB}$. This can be attributed to the loss in manufacturing. Limitations for UWB signals are $500 \mathrm{MHz}$ bandwidth or FBW greater than $20 \%$ (Chu, 2016). A $3.58 \mathrm{GHz}$ bandwidth for the LP region is observed from 0 to $3.58 \mathrm{GHz}$. The central frequency is $1.79 \mathrm{GHz}$ and the $\mathrm{FBW}$ is $50 \%$ (Equation 6). For the BP region, which is observed from 14.38 to 
$21.65 \mathrm{GHz}$, the bandwidth is $7.27 \mathrm{GHz}$. The central frequency is $18.02 \mathrm{GHz}$ and the FBW is $40.34 \%$. Therefore, for both passband regions, an ultra-wideband is observed. Furthermore, there is also a UWB rejection band $(<10 \mathrm{~dB})$ in the frequency range from 4.46 to $14.07 \mathrm{GHz}$ according to measurement results. Figure 5 shows the frequency response of the proposed SIR filter according to simulation and measurement results.

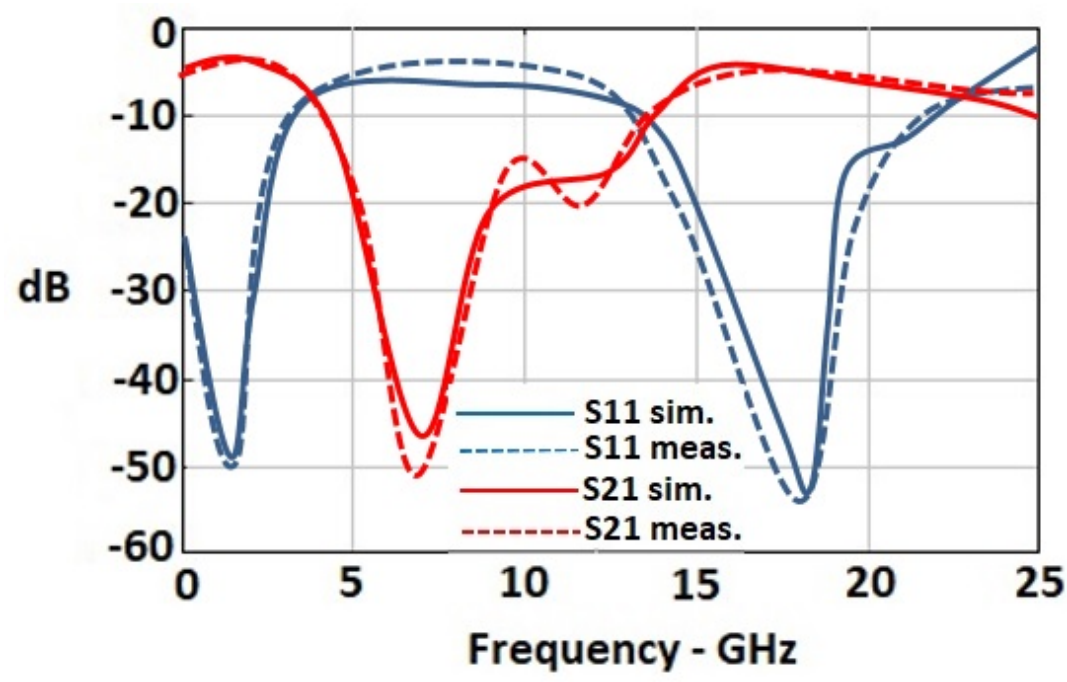

Figure 5. Frequency response of the proposed SIR Filter.

\section{CONCLUSIONS}

Two new microwave filter configurations for high frequency and UWB applications are introduced. Firstly, a multiband BSF using four open stubs and fractal structure has been proposed, simulated, and fabricated. The simulation is carried out in the range of $0.1 \mathrm{GHz}$ to $13 \mathrm{GHz}$, where the signals are rejected at two central frequencies $(3.75 \mathrm{GHz}$ and $9.75 \mathrm{GHz})$ and the others are allowed to pass. Three passing regions are formed (low-pass, band-pass, and high-pass), achieving the FBW of $66.67 \%$ in the band-pass region. The attenuation is well achieved reaching $-44.6 \mathrm{~dB}$ with a $\% 40 \mathrm{FBW}$. The filter met the requirements of high performance, low losses, compact size of approximately $17 \mathrm{~mm}$ x $17 \mathrm{~mm}$, and affordable price. The open stub method ensured the three passband regions, while fractal structure ensured the smooth response and small box size. The advantages of the proposed filter over other some relevant studies have been confirmed by a comparative table. Secondly, a UWB filter design implementing SIR and modified H-shaped structure is presented. The filter design is achieved by observing a wide frequency range from 0 to $25 \mathrm{GHz}$. This newly designed filter exhibits a solid performance with virtually no pass-band loss, and a passband return-loss constantly over $20 \mathrm{~dB}$. Furthermore, according to the measurement results, a rejection band of at least $10 \mathrm{~dB}$ is obtained between $4.46 \mathrm{GHz}$ and $14.7 \mathrm{GHz}$. Both of the proposed filters are very compact and utilize a low-cost fabrication material, which highly reduces its production costs. Furthermore, it has been shown that the cut-off frequencies of this filters can be modified easily by adjusting certain dimensions allowing possibility for the future filter design considerations. 


\section{ACKNOWLEDGMENT}

The authors would like to thank Tamara Electronic-Istanbul, for the fabrication, and Associate Professor Dr. Hamid Torpi from the Yildiz Technical University-Istanbul, for the measurements.

\section{REFERENCES}

Cameron, R. J., Kudsia, C. M., \& Mansour, R. R. 2018. Microwave filters for communication systems: fundamentals, design, and applications. John Wiley \& Sons.

Chen, X. 2021. Synthesis of multi-band filters based on multi-prototype transformation. IET Microwaves, Antennas \& Propagation 15(2): 103-114.

Chu, J. 2016. An Introduction to UWB Antenna Design. IEEE Microwave Magazine 17(3): 54-55.

Chu, P., Guo, L., Zhang, L., \& Wu, K. 2019. Wide Stopband Band Pass Filter Implemented by Stepped Impedance Resonator and Multiple In-Resonator Open Stubs. IEEE Access 7: 140631-140636.

Crnojević-Bengin, V. 2015. Advances in multi-band microstrip filters. Cambridge University Press.

Guyette, A. C., \&Naglich, E. J. 2016. Short-through-line band stop filters using dual-coupled resonators. IEEE Transactions on Microwave Theory and Techniques 64(2): 459-466.

Hua, C., \& Lu, Y. 2018. Compact UWB band pass filter with a reconfigurable notched band. International Journal of RF and Microwave Computer-Aided Engineering 28(4): e21212.

Hung, S. Y., Li, G. P., \&Guo, Y. 2020. Compact customisablebandstop-bandpass-bandstop cascaded filter based on substrate integrated waveguide coax cavities. IET Microwaves, Antennas \& Propagation 14(4): 281-292.

Karpuz, C., Gorur, A. K., \&Emur, M. 2016. Quad-band microstrip band stop filter design using dual-mode open loop resonators having thin film capacitors. IEEE Microwave and Wireless Components Letters26(11): 873-875.

Koirala, G. R., Shrestha, B., \& Kim, N. Y. 2016. Compact dual-wideband band stop filter using a stubenclosed stepped-impedance resonator. AEU-International Journal of Electronics and Communications70(2): 198-203.

Lan, S. W., Weng, M. H., Hung, C. Y., \& Chang, S. J. 2016. Design of a compact ultra-wideband bandpass filter with an extremely broad stopband region. IEEE Microwave and Wireless Components Letters 26(6): 392-394.

Majidifar, S., \& Hayati, M. 2017. New approach to design a compact triband band pass filter using a multilayer structure. Turkish Journal of Electrical Engineering \& Computer Sciences25(5): 4006-4012.

Martín, F., Zhu, L., Hong, J. S., \& Medina, F. 2018. Balanced microwave filters. John Wiley \& Sons, Incorporated.

Naglich, E. J., \&Guyette, A. C. 2015. Reflection-mode band stop filters with minimum through-line length. IEEE Transactions on Microwave Theory and Techniques 63(10): 3479-3486.

Ren, B., Liu, H., Ma, Z., Ohira, M., Wen, P., Wang, X., \& Guan, X. 2018. Compact dual-band differential bandpass filter using quadruple-mode stepped-impedance square ring loaded resonators. IEEE Access 6: 21850-21858.

Sarkar, P., Pal, M., \&Ghatak, R. 2016. A compact dual stopband band stop filter using defected SIR and Hilbert shape fractal structure. Microwave and Optical Technology Letters58(6): 1345-1347. 
Shrestha, B., \& Kim, N. Y. 2015. Microstrip wideband band stop filter with open stubs for UWB applications. Microwave and Optical Technology Letters 57(4): 1003-1006.

Sirci, S., Sánchez-Soriano, M. Á., Martínez, J. D., \&Boria, V. E. 2019. Advanced filtering solutions in coaxial SIW technology based on singlets, cascaded singlets, and doublets. IEEE Access 7: 29901-29915.

Şahin, E. G., Gorur, A. K., Karpuz, C., \&Gorur, A. 2016. Design of UWB microstrip band pass filter using stub-loaded quintuple-mode resonator. Microwave and Optical Technology Letters58(3): 662-666.

Tütüncü, B. 2020. Mutual coupling reduction using coupling matrix-based band stop filter. AEU-International Journal of Electronics and Communications 124: 153342.

Wei, F., Qin, P. Y., Guo, Y. J., Ding, C., \& Shi, X. W. 2016. Compact balanced dual-and tri-band BPFs based on coupled complementary split-ring resonators (C-CSRR). IEEE Microwave and Wireless Components Letters 26(2): 107-109.

Xia, X., Chen, F., Cheng, X., \& Deng, X. 2018. A compact ultra-wideband band pass filter with good selectivity based on interdigital coupled-line. International Journal of RF and Microwave Computer-Aided Engineering28(9): e21419.

Xu, J., Bi, K., Zhai, X., Hao, Y., \&Mcdonald-Maier, K. D. 2019. A dual-band microwave filter design for modern wireless communication systems. IEEE Access 7: 98786-98791.

Yang, Q., Jiao, Y. C., \& Zhang, Z. 2018. Compact multiband band pass filter using low-pass filter combined with open stub-loaded shorted stub. IEEE Transactions on Microwave Theory and Techniques 66(4): 1926-1938.

Zhang, X. C., Chen, X., Sun, L., Huang, Y. S., \& Gao, X. F. 2019. A Microstrip Stepped-Impedance Resonator Bandpass Filter Based on Inductive Coupling. Frequenz 73(1-2): 7-11.

Zobeyri, M. R., \&Eskandari, A. R. 2018. Design of single-and dual-band BPFs using folded $0^{\circ}$ feed structures and embedded resonators. AEU-International Journal of Electronics and Communications 96: 18-29.

Zuo, X., \& Yu, J. 2017. Miniaturized planar coupled-line band stop filter with improved and extended pass-band performances. Microwave and Optical Technology Letters 59(9): 2260-2262. 